\title{
KOMPLEX, ABER NICHT KOMPLIZIERT: INTERDISZIPLINÄRE THERAPIE PRIMÄRER KOPFSCHMERZEN
}

Katrin Lengnick, Andrea Koller

Kopfschmerzen sind ein häufiger Vorstellungsgrund in der kinderärztlichen Praxis und haben eine hohe Chronifizierungsrate bis ins Erwachsenenalter. ${ }^{1)} \mathrm{Da}$ her ist es wichtig, diese frühzeitig ernst zu nehmen und rechtzeitig effektiv zu behandeln.

Eine effektive Behandlung umfasst neben der adäquaten Diagnostik und medikamentösen Therapie auch die Edukation sowie die Unterstützung in der Behandlungsumsetzung im Alltag.

Die multifaktorielle Entstehung und Aufrechterhaltung von Kopfschmerzen ist mittlerweile gut belegt.1) Dies bedingt einen multimodalen Behandlungsansatz, welcher der Komplexität der Entstehung und Aufrechterhaltung Rechnung trägt. ${ }^{2)}$ Dabei ist das primäre Ziel der Kopfschmerzbehandlung, neben der Verbesserung der Schmerzsituation, die Besserung der Lebensqualität und der Funktionalität im Alltag.

Dies ist sinngemäss nach den neuen Therapieempfehlungen der SKG (Schweizerische Kopfwehgesellschaft) ${ }^{3)}$ durch folgendes Management zu erreichen:

1) Rasche Diagnoseklärung

2) Akzeptanz der Diagnose durch Edukation

3) Patientenzentrierte Ressourcenaktivierung

4) Erstellen eines gemeinsamen Behandlungsplans

5) Wertschätzende Abklärung teilweise ausrechend, ohne weitere Behandlungsnotwendigkeit

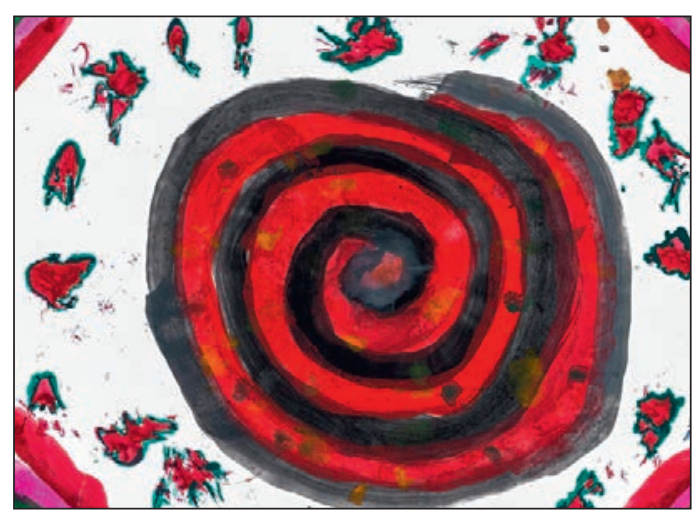

Abbildung 1. Dolographie Fabio 10 Jahre, Spannungskopfschmerzen

\section{Rasche Diagnosestellung}

Das Ziel einer raschen Diagnosestellung ist es, schnellstmöglich Klarheit und Sicherheit zu schaffen und Ängste zu reduzieren. Dafür ist es essentiell, möglichst frühzeitig das individuelle Störungsmodell von Patient und Familie zu erfassen und sekundäre Ursachen mittels gründlicher Anamnese und klinischer Untersuchung, sowie ggf. weiterführender Diagnostik auszuschliessen. Die klare Benennung der Diagnose gemäss IHS Kriterien für primäre Kopfschmerzen (siehe Tabelle 1) stellt einen wichtigen Schritt in der Aufklärung von Kind und Eltern dar. Anamnestisch kann neben dem Kopfschmerztagebuch, sowie der Sprechstunde vorausgehende Fragebogen zu familiären, schulischen und sozialen «Auslösern» und «aufrechterhaltenden Faktoren», auch eine Dolographie hilfreich sein (siehe Abbildung 1 und 2). Bei Auftreten von (neuro-) pädiatrischen Warnzeichen und/oder häufigen, sozial stark einschränkenden Kopfschmerzen kann die zeitnahe Aufgleisung einer weiterführenden Diagnostik (siehe 1.2.) notwendig werden.

\subsection{Charakteristika primärer Kopfschmerzen im Kindesalter}

\subsubsection{Migräne}

Bei der Migräne im Kindes- und Jugendalter (10-20\%) 1) treten episodisch akute Kopfschmerzen mittlerer bis hoher Intensität auf, welche zu einer Unterbrechung der aktuellen Tätigkeit führen. In 60-80\% handelt es

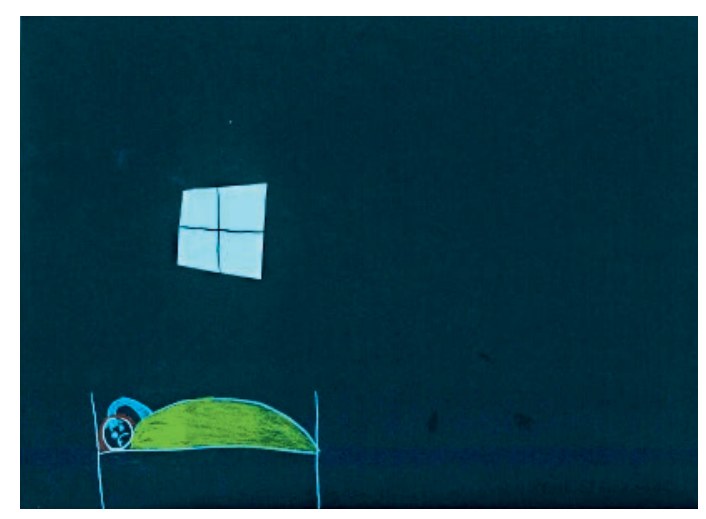

Abbildung 2. Dolographie Leandra 8 Jahre, Migräne

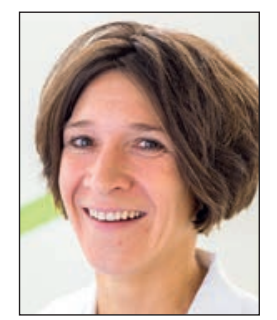

Katrin Lengnick

https://doi.org/ $10.35190 / \mathrm{d} 2021.4 .8$
Korrespondenz: Katrin. Lengnick-Lampadius @kispisg.ch 


\section{Fortbildung}

sich im Kindes- und Jugendalter um Migräne ohne Aura. Häufig können Kinder jedoch über autonome, psychische oder neurologische Vorläufersymptome berichten ${ }^{5}$ ehe der Migränekopfschmerz oft in den frühen Morgenstunden beginnt. Dabei handelt es sich ursächlich um eine neurogene Entzündung im trigeminovaskulären System, welche sowohl durch erbliche Disposition als auch durch Umweltfaktoren beeinflusst wird. ${ }^{4)}$

\subsubsection{Spannungskopfschmerz}

Spannungskopfschmerzen (60-80\%) können durch eine erhöhte periphere (muskuläre Anspannung) und/oder zentrale (Stress) Aktivierung ausgelöst und verstärkt werden. ${ }^{4)}$ Sie treten episodisch oder chronisch auf, sind von leichter bis mittlerer Intensität, führen nicht zur Unterbrechung der Tätigkeit und lassen sich durch Ablenkung und körperliche Bewegung verbessern.

\subsubsection{Kopfschmerzen vom Mischtyp}

Bei $50-60 \%$ aller Patienten mit Migräne zeigt sich ein Mischbild mit zusätzlichem Auftreten von Spannungskopfschmerzen. ${ }^{5)}$ Auch wenn Prädisposition und Auslöser beider Kopfschmerzarten überlappend sein können, kommt der Unterscheidung ein hoher Stellenwert zu, da sich die Therapie der jeweiligen Kopfschmerzarten massgeblich unterscheidet (siehe Tabelle 1+3).

\subsection{Weiterführende Diagnostik}

Die Diagnose primärer Kopfschmerzen ist eine klinische Diagnose, die anhand einer sorgfältigen Anamnese und kinderneurologischen Untersuchung sowie Verlaufsbeurteilung gestellt werden kann ${ }^{6}$ Bestehen atypische Symptome und/oder Warnzeichen ist entsprechend der möglichen Differentialdiagnosen eine weiterführende Abklärung indiziert, wobei die MRT-Bildgebung an erster Stelle steht.7) Je nach Störungsverständnis und Ängsten im familiären System, ist in ausgewählten Fällen auch ohne klassische «red flags» eine kraniale Bildgebung sinnvoll, um im Verlauf zu einem gegenseitig akzeptierten Behandlungsplan zu kommen und eine rein somatische Fixierung aufzulösen. Bei psychosozialen Warnsignalen wie Schulabsentismus, schulischer Leistungsabfall, sozialer Rückzug, Gefühlsund Verhaltensveränderungen, sowie bei offen geäussertem oder indirekt spürbarem Widerstand gegenüber fachärztlichen Veränderungsvorschlägen («Ja, aber...») ist über eine weiterführende neuropsychologische und/oder kinder- und jugendpsychiatrische Diagnostik nachzudenken. Hier können sowohl primäre psychische Erkrankungen (z.B. depressive Verstimmung) als auch kognitive Überforderungen und psychologische Zielkonflikte (z.B. Befürchtung: Schmerzreduktion führt zu geringerer Zuwendung) erfasst werden.

\section{3. «Red flags»}

- fokal-neurologische Symptome (Augenbewegungsstörung, sensible / motorische Ausfälle, Ataxie, Stauungspapille)

- systemische Zeichen (Nüchternerbrechen, Fieber, Veränderung von Schlaf, Appetit, Verhalten, Bewusstsein)

- ungewöhnliche Intensität, Dauer der Kopfschmerzen, Progredienz, fehlende FA

- Alter $<3$ (-5) Jahre

\section{Akzeptanz der Diagnose durch Edukation}

Edukation ist ein elementarer Baustein in der Therapie von primären pädiatrischen Kopfschmerzen als Basis für das Verständnis der weiteren Behandlungsinhalte und -schritte. Mit der auf die Familie abgestimmten Wissensvermittlung wird die Grundlage für ein gemeinsames Störungsmodell im Sinne des bio-psycho-sozialen Modells gelegt, was wiederum die Motivation für die angestrebten Verhaltensänderungen stärkt. Dabei ist die Wirksamkeit von Schmerzedukation bei erwachsenen Migräne-Patienten ${ }^{8)}$ und bei Kindern mit chronischen Schmerzen") bereits gut

\begin{tabular}{l|l|l} 
Merkmale & Migräne & Spannungskopfschmerzen \\
\hline Häufigkeit & gelegentliche Anfälle & bis zu täglich \\
\hline Dauer & 2-72 Stunden & 30 Min. bis mehrere Tage \\
\hline Intensität & mittel bis schwer & leicht bis mittel \\
\hline Charakter & $\begin{array}{l}\text { meist einseitig pulsierend } \\
\text { (bei Kindern häufig bifrontal } \\
\text { oder holokran) }\end{array}$ & beidseitig drückend \\
\hline Übelkeit/Erbrechen & häufig & selten \\
\hline Reizempfindlichkeit & stark (Photo-/Phonophobie) & gering \\
\hline Körperliche Aktivität & verstärkt die Kopfschmerzen & $\begin{array}{l}\text { keine Verstärkung, } \\
\text { wirkt eher lindernd }\end{array}$ \\
\hline Ablenkung vom Schmerz & kaum möglich & gut möglich \\
\hline
\end{tabular}

Tabelle 1. Diagnosekriterien gemäss IHS für episodische Spannungskopfschmerzen und Migräne ohne Aura im Kindes- und Jugendalter 


\section{Fortbildung}

belegt und führt nachweislich zu einer Abnahme der Schmerzhäufigkeit und der schmerzbedingten Beeinträchtigung, bei steigender Lebensqualität ${ }^{8,9)}$

Kernelemente der Edukation bei primären Kopfschmerzen sind $d^{5)}$ :

- Aufklärung über Kopfschmerz-begünstigende Faktoren und deren Beeinflussbarkeit (bio-psycho-soziales Modell, Lifestyle-Massnahmen)

- Theoretische (Pathophysiologie) und praktische Differenzierung zwischen Spannungskopfschmerz und Migräne (z.B. Schmerzverstärkung durch Hüpfen bei Migräne)

- frühzeitige Identifizierung einer Migräneattacke und korrekte medikamentöse Akutbehandlung

Bei chronifizierten Kopfschmerzen kann zu einem späteren Zeitpunkt auf Gründe für die Aufrechterhaltung von Schmerzen, den Schmerzkreislauf und das «Alarmsystem Schmerz» eingegangen werden.

Da eine spielerische Wissensvermittlung bei Kindern unabdingbar ist, kommen zur Edukation neben Bildmaterial und Buchtipps (z.B. «Rote Karte für den Schmerz») auch YouTube Videos und Edukations-Apps (z.B. «Migräne hab ich im Griff» und «Applaus-Projekt») zum Einsatz (siehe Tabelle 2).

\section{Ressourcenaktivierung}

Alltagsrelevante Lifestyle Massnahmen, wie regelmässige ausgewogene Mahlzeiten, Schlafrhythmus, Bewegung, Trinkmenge (Wasser) und ausreichend unverplante Freizeit sind im Kindesalter häufig hilfreich. Hinzu kommt zu evaluieren, ob Überbelastungen durch übermässigen Medienkonsum, Überforderung in der Schule, soziale Ausgrenzung, Freizeitüberangebot oder familiäre Stressoren vorliegen.1.3) Ein wichtiger, rasch zu erfassender Faktor ist das Vorliegen häufiger Schul-/Lehr-Absenzen, da dies einen zunehmenden Druck auf das familiäre System und konsekutiv das Risiko einer inadäquaten, oft invasiven Diagnostik und Therapie und einem erhöhten Risiko für eine Chronifizierung der Symptomatik zur Folge haben kann. ${ }^{10)}$

Im Sinne des lösungsorientierten Ansatzes ist es wichtig, auf Ressourcen und Ziele von Kindern und Eltern zu fokussieren, und dabei Ausnahmen, die Beeinflussbarkeit der Kopfschmerzen und aktive Bewältigungsstrategien, welche sich als bisher nützlich erwiesen haben besonders hervorzuheben. Dadurch kann die Motivation zur Mitarbeit und das Gefühl von Selbstwirksamkeit, aber auch die Eigenverantwortung bei den Kindern geweckt und die selbstempfundene Hilflosigkeit reduziert werden. Ziel soll sein, dass Patient und Eltern die Sprechstunde mit konkreten Ideen verlassen, was sie präventiv und in der Akutsituation tun können, um die Schmerzsituation positiv zu beeinflussen.
Das Wiedererlangen der schulischen und sozialen Funktionsfähigkeit hat dabei eine hohe Priorität. Durch maladaptive Bewältigungsstrategien (übermässiges Vermeidungs- und Schonverhalten, Schulabsenz, Einnahme von Medikamenten trotz fehlender Wirksamkeit), basierend auf (gutgemeintem) falschem Wissen, können die schmerzbedingte Beeinträchtigung, die emotionale Belastung, der Stress und die somatischen Symptome rasch zunehmen und eine Chronifizierung begünstigen. ${ }^{11)}$ Dies auch dadurch, dass immer mehr Aktivitäten reduziert werden, die Freude und soziale Teilhabe sowie Selbstwertstärkung ermöglichen. Durch aktive Bewältigungsstrategien (Ablenkung, Pausenmanagement) und das schrittweise Heranführen an korrigierende Bewältigungserfahrungen (z.B. Kopfweh und Müdigkeit am Morgen verschlechtern sich nicht durch Schulbesuch) kann die Zuversicht in die eigene Wirksamkeit wiedererlangt und die Lebensqualität gesteigert werden. Dies hat einen positiven Effekt auf die Alltagsbeeinträchtigung und die Schmerzintensität.

\section{Gemeinsamer multimodaler Behandlungsplan}

Bei der Behandlung primärer Kopfschmerzen empfiehlt sich aufgrund der Mehrdimensionalität der Ursache primärer Kopfschmerzen ein multimodales Therapieangebot. Dabei soll mit Patienten und Familie geklärt werden, was es im Sinne des bio-psycho-sozialen Schmerzmodells braucht, um die Lebensqualität in der aktuellen Situation zu verbessern, was die konkreten nächsten Umsetzungsschritte sind, und ob ein Einbezug der Schule oder einer psychologischen Begleitung sinnvoll ist. Das Zusammenspiel von Selbstmanagement, akuter sowie präventiver Behandlung hat sich dabei als effektiv erwiesen.

\subsection{Akute Spannungskopfschmerzen - Motto «defensiv»"6)}

Im Vordergrund stehen nicht-medikamentöse Massnahmen (siehe Tab. 2). Nur in Ausnahmefällen sollten NSAR eingesetzt werden, da sonst bei häufigen Episoden das Risiko des Übergebrauchskopfschmerz besteht.

\subsection{Akute Migräneepisode -}

Motto «offensiv»")

Hier gilt es frühzeitig und richtig dosiert («hit fast, hit hard») ein gut wirksames und sicheres Medikament einzunehmen (siehe Tabelle 3). Die genaue Erläuterung der Wirksamkeit, inklusive Zeit bis zum Wirkeintritt, Wirkdauer und Nebenwirkungen sind dabei wichtige Faktoren, um die Compliance und die Effektivität der Medikation zu erhöhen. Durch eine effektive Attacken-Therapie kann das Gefühl des Kontrollverlustes reduziert und der allgemeine Umgang mit den Schmerzen verbessert werden.

Empfohlen werden primär NSAR (Mittel der 1. Wahl). Bei unzureichender Wirksamkeit und ab dem 12. Lebensjahr ist auch die alleinige oder kombinierte Gabe mit Triptanen möglich (siehe Tabelle 3). Dies 
Fortbildung

\begin{tabular}{|c|c|c|}
\hline Verfahren & Inhalt & Tipps zur Umsetzung \\
\hline Psychoedukation & $\begin{array}{l}\text { - begünstigende Faktoren und } \\
\text { zugrundeliegende } \\
\text { Mechanismen } \\
\text { - Erfahrung d. Patienten bzgl. } \\
\text { Ausnahmen und Beeinflussbar- } \\
\text { keit } \\
\text { - Bio-psycho-soziales Modell } \\
\text { - Differenzierung Migräne vs } \\
\text { Spannungskopfschmerz } \\
\text { - Übergebrauchskopfschmerz } \\
\text { - Ansatzpunkte für Therapie und } \\
\text { Verhaltensänderung }\end{array}$ & $\begin{array}{l}\text { Online Comics «Migräne hab ich im } \\
\text { Griff» «Mütze hat den Kopfschmerz } \\
\text { satt» «Den Schmerz verstehen und } \\
\text { was zu tun ist in } 10 \text { Minuten» } \\
\text { App fürs Smartphone mit Eduka- } \\
\text { tionsmaterial «Applaus-Projekt», } \\
\text { «PainT» } \\
\text { Nachfragen: Was hat die Familie } \\
\text { verstanden? Was können sie für sich an } \\
\text { hilfreichen Informationen mitnehmen? }\end{array}$ \\
\hline $\begin{array}{l}\text { Schmerzbewältigung/ } \\
\text { Coaching }\end{array}$ & $\begin{array}{l}\text { Aufbau aktiver Bewältigungsstra- } \\
\text { tegien, Pausenmanagement, } \\
\text { korrigierender Erfahrungen, } \\
\text { präventiver Verhaltensanpassung } \\
\text { - Etablierung im Alltag }\end{array}$ & $\begin{array}{l}\text { Ablenkung (Kopf und Körper auf } \\
\text { andere Gedanken bringen) durch } \\
\text { freudvolle Tätigkeiten und körperlicher } \\
\text { Gegenreiz (Kühlen, Pfefferminzöl, } \\
\text { Akupressur, Sport und Bewegung) } \\
\text { Nachfragen: Wo sieht die Familie } \\
\text { mögliche Stolpersteine in der } \\
\text { Umsetzung? }\end{array}$ \\
\hline Entspannungsverfahren & $\begin{array}{l}\text { Progressive Muskelrelaxation } \\
\text { (PMR), autogenes Training, } \\
\text { Bauchatmung, Imaginative } \\
\text { Verfahren }\end{array}$ & $\begin{array}{l}\text { Entspannungsmomente } \\
\text { im Alltag Büchertipps: «Chillen un- } \\
\text { term Sorgenbaum», «Geschich- } \\
\text { ten von der Fly», «Kapitän Nemo } \\
\text { Geschichten», «Die Ruhe im Was- } \\
\text { serglas», } \\
\text { Schmerz transformieren bzgl. Farbe, } \\
\text { Form oder eigener Distanz }\end{array}$ \\
\hline Biofeedback & $\begin{array}{l}\text { Rückmeldung von Körperfunktio- } \\
\text { nen, um den Schmerzzustand } \\
\text { positiv zu beeinflussen (Atmung, } \\
\text { Tonus) }\end{array}$ & $\begin{array}{l}\text { Zahlreiche YouTube-Filme und Apps } \\
\text { fürs Smartphone zur freien Verfügung }\end{array}$ \\
\hline $\begin{array}{l}\text { Operante } \\
\text { Schmerztherapie }\end{array}$ & $\begin{array}{l}\text { - Trigger Management } \\
\text { - Veränderung schmerzaufrecht- } \\
\text { erhaltender Aspekte in Familie, } \\
\text { Schule, Lebensführung }\end{array}$ & $\begin{array}{l}\text { Ressourcentagebuch Elterncoaching } \\
\text { Alltagstipps für Kinder und Eltern } \\
\text { schmerzunabhängige Zuwendung } \\
\text { Verstärkerplan «gut bewältigte Tage» }\end{array}$ \\
\hline $\begin{array}{l}\text { Kognitive } \\
\text { Verhaltenstherapie }\end{array}$ & $\begin{array}{l}\text { Änderung dysfunktionaler Ge- } \\
\text { wohnheiten, Gedanken Belas- } \\
\text { tungs-/Stressmanagement } \\
\text { Emotions- und Spannungsregu- } \\
\text { lation }\end{array}$ & $\begin{array}{l}\text { Schmerz als Regulation (Alternativen?) } \\
\text { Vermeidung vermeiden Einbezug } \\
\text { weiterer wichtiger Personen } \\
\text { (z.B. Vernetzung Schule-Eltern) }\end{array}$ \\
\hline
\end{tabular}

Tabelle 2. Behandlungsbausteine zur nichtmedikamentösen Therapie primärer Kopfschmerzen im Kindesalter

kann nötig werden, um einerseits den Wirkeintritt zu verkürzen (frühmorgendliche Migräne) und andererseits ein Wiederauftreten der Migräne (Rebound Kopfschmerz) zu verhindern.

Bei suffizienter Schmerztherapie ist häufig keine antiemetische Therapie mehr notwendig. Sollte aber das Erbrechen im Vordergrund stehen (z.B. zyklisches Erbrechen), ist eine solche z.B. mit Ondansetron, Dopamin-A2 Antagonisten (Cave: Dystonie) oder Aprepitant zu erwägen.

Der Status migränosus (Dauer $\geq 72 \mathrm{~h}$ ) ist ein neuropädiatrischer Notfall und Bedarf der Zuweisung und Behandlung im stationären Setting.

\subsection{Chronische Kopfschmerzen -}

Motto «interdisziplinär \& multimodal»

Bei chronischen Kopfschmerzen (Schmerzen an mehr als 15 Tagen/Monat, seit mehr als 3 Monate) scheint es im ZNS einerseits zu einer Blockierung antinozizeptiver Systeme und andererseits zu einer Schmerzsensibilisierung zu kommen. ${ }^{4)}$ Daher stehen bei der Behandlung die nicht-medikamentösen Behandlungsverfahren (siehe Tabelle 2) im Vordergrund. In der Pädiatrie werden daher Massnahmen zur Anpassung des Lifestyles, sowie psychologische Intervention der medikamentösen Prophylaxe vorgezogen. ${ }^{6,16)}$ Durch sie können Stress und Anspannung sowie schmerzbezogene Ängste abgebaut und Entspannung, Selbstwirksamkeit, Bewegung und Freude aufgebaut werden. ${ }^{13)}$ Eine wichtige Rolle kommt hier auch der Stärkung der 


\section{Fortbildung}

\begin{tabular}{l|l|l|l|l|l} 
Wirkstoff & Stellenwert & Dosierung & Intervall & Wirkmaximum & Kommentar \\
\hline Ibuprofen & $\begin{array}{l}\text { Mittel der 1. Wahl bei } \\
\text { Migräne, (SKS) }\end{array}$ & $10-15 \mathrm{mg} / \mathrm{kg}$ & $6 \mathrm{~h}$ & $1-2 \mathrm{~h}$ & $\begin{array}{l}\text { ggf. Schmelztabletten KI: } \\
\text { Nierenerkrankung }\end{array}$ \\
\hline Paracetamol & $\begin{array}{l}\text { Alternative bei Mig- } \\
\text { räne, (SKS) }\end{array}$ & $10-15 \mathrm{mg} / \mathrm{kg}$ & $6 \mathrm{~h}$ & $30-60 \mathrm{~min}$ & $\begin{array}{l}\text { variable Bioverfügbarkeit } \\
\text { NW: hepatotoxisch }\end{array}$ \\
\hline Sumatriptan & $\begin{array}{l}\text { Bei Migräne } \\
\text { ab 12 Jahren }\end{array}$ & $\begin{array}{l}10 \mathrm{mg}(<40 \mathrm{~kg}) \\
20 \mathrm{mg}(>40 \mathrm{~kg})\end{array}$ & $12 \mathrm{~h}$ & $\begin{array}{l}15 \mathrm{~min}(\text { nasal) } \\
30 \mathrm{~min}(\text { p.o. })\end{array}$ & $\begin{array}{l}\text { nasal oder p.o. NW: } \\
\text { Enge- und Kältegefühl }\end{array}$ \\
\hline Zolmitriptan & $\begin{array}{l}\text { Alternative zu } \\
\text { Sumatriptan }\end{array}$ & $\begin{array}{l}2,5 \mathrm{mg}(<40 \mathrm{~kg}) \\
5 \mathrm{mg}(>40 \mathrm{~kg})\end{array}$ & $12 \mathrm{~h}$ & $\begin{array}{l}15 \mathrm{~min}(\text { nasal) } \\
30 \mathrm{~min}(\text { p.o. })\end{array}$ & «Off-label» \\
\hline
\end{tabular}

Tabelle 3. Akutmedikation bei primären Kopfschmerzen im Kindesalter ${ }^{3)}$ SKS=Spannungskopfschmerzen

Eltern zu, welche häufig sehr verunsichert sind, jedoch durch richtige Instruktion den Therapieerfolg durch Unterstützung einer aktiven Bewältigungsstrategie massgeblich positiv beeinflussen können. ${ }^{11}$

Gründe für den Einsatz einer zusätzlichen medikamentösen Migräneprophylaxe im Kindesalter (siehe Tabelle 4) im Rahmen der multimodalen Therapie können hohe Attackenfrequenz, hemiplegische Migräne, Übergebrauchs-Kopfschmerz sowie eine deutliche Einschränkung der Lebensqualität und Funktionalität im Alltag sein. Da für kein Medikament zur prophylaktischen Behandlung der kindlichen Migräne eine spezifische Zulassung besteht, sollen diese Medikamente aufgrund ihres oft erheblichen Nebenwirkungsprofils äusserst restriktiv eingesetzt werden. ${ }^{12}$ Z Zudem ist zu berücksichtigen, dass durch den Einsatz prophylaktischer Medikamente, die Chronifizierung, insbesondere bei gleichzeitiger Vernachlässigung psychologischer Interventionen, verstärkt werden kann.

Aufgrund des «parents beliefs modell» und des hohen Placeboeffektes jeglicher Therapie von Kopfschmerzen im Kindes- und Jugendalter ${ }^{14}$, kommen auch physiotherapeutischen Interventionen, der Neurostimulation, sowie komplementärmedizinischen
Therapieansätzen eine Bedeutung zu. Voraussetzung hierfür ist jedoch immer die Eigenmotivation der Patienten, sowie die Möglichkeit zum selbstwirksamen Steuern und Handeln. Mögliche Optionen hierfür sind z.B. triggerpunktspezifische Physiotherapie, Akupressur, TENS, Hypnotherapie sowie tiergestützte Therapien.

\section{Nicht in jedem Fall besteht nach wertschätzender Abklärung Behandlungsnotwendigkeit}

Das bedeutet für den Behandler, dass er im Sinne des bio-psycho-sozialen Modells eine Diagnose stellt und zusammen mit dem Patienten eine möglichst passende Behandlungsstrategie erarbeitet, welche diesen befähigt, die Verantwortung für seine (Schmerz-) Situation zu übernehmen. «Niemand kann für seinen Schmerz verantwortlich gemacht werden. Aber der Versuch, die Verantwortung für ihn zu übernehmen, ist eine der wirkungsvollsten Strategien im Umgang mit ihm».15)

Für das Literaturverzeichnis verweisen wir auf unsere Online Version des Artikels.

\begin{tabular}{l|l|l} 
Wirkstoff & Dosierung & Kommentar \\
\hline Magnesium & $\begin{array}{l}9 \mathrm{mg} \text { oder } 0,5 \mathrm{mmol} / \mathrm{kg} / \mathrm{d} \\
\text { in 2 ED über 3 Monate }\end{array}$ & $\begin{array}{l}\text { NW: Bauchschmerzen, Blähungen, } \\
\text { Durchfall }\end{array}$ \\
\hline Riboflavin & $\begin{array}{l}10 \mathrm{mg} / \mathrm{kg} / \mathrm{d} \text { oder 200-400mg } \\
\text { in 2 ED über 3 Monate }\end{array}$ & NW: Urin - Gelbfärbung \\
\hline Flunarizin & $\begin{array}{l}5 \mathrm{mg}(<40 \mathrm{~kg}) \\
10 \mathrm{mg}(>40 \mathrm{~kg})\end{array}$ & $\begin{array}{l}\text { NW: Gewichtszunahme, Übelkeit, } \\
\text { Müdigkeit, Depressivität, extrapy- } \\
\text { ramidale Störungen }\end{array}$ \\
\hline
\end{tabular}

Tabelle 4. Medikamentöse Migräne- Prophylaxe4)

\footnotetext{
Autorinen

Dr. med. Katrin Lengnick, Zentrum für Kinderneurologie, Entwicklung und Rehabilitation (KER-Zentrum), Fachbereich Neuropädiatrie, Ostschweizer Kinderspital, St. Gallen Lic. phil. Andrea Koller, Neuropsychologie im Zentrum für Kinderneurologie, Entwicklung und Rehabilitation, St. Gallen

Die Autorinen haben keine finanziellen oder persönlichen Verbindungen im Zusammenhang mit diesem Beitrag deklariert.
} 